\title{
Driver Assistance System Based on Vehicular Communication
}

\author{
Somaskandan $M^{1}$, Avinash $B^{2}$, Sanjay Raj $D^{3}$, Someshwar $G^{4}$, Vignesh $M^{5}$ \\ Assistant Professor, Department of Information Technology, Panimalar Engineering College, Chennai, India ${ }^{1}$ \\ Student, Department of Information Technology, Panimalar Engineering College, Chennai, India $2,3,4,5$
}

\begin{abstract}
Vehicular communication system is a system which can guarantee the safer transport by enabling vehicles to communicate with one another, and their surroundings and thereby it can provide driver assistance. The proposed system is capable of measuring the level of fuel in the tank and send enquiry to know the nearest location of fuel station, then wireless communication equipped fuel station automatically gives the information about that location. This system enables immediate payment system in which the vehicle can pay the tolls in arrears by the wireless fund transfer method. This system is also capable of detecting an approaching landslide or occurring one and transmitting pre safe warning. In addition to this, the system enables us to automatically monitor and/or control the actual speed of a vehicle relative to the legal speed limit of the geographic area the vehicle is located in. By implementing this effective and reliable system the road accident can be reduced and road safety will be improved. If this idea comes to the real life it will be more useful for the people as well the government since it take lots of measures to reduce the accidents.
\end{abstract}

Keywords: level of fuel, immediate payment, pre safe warning, legal speed limit.

\section{INTRODUCTION}

A. Need

According to the report provided by the Supreme Court Committee on Road Safety 1,46,377 lives lost on the roads in the year of 2017 till September in India. To safeguard the valuable lives of people we proposed a system called "Driver Assistance System Based on Vehicular Communication". Vehicular communication is thought to have great potential for improving road safety. The vehicular communication system is a system which can guarantee the safer transport by enabling vehicles to communicate with one another, and their surroundings and thereby it can provide assistance to the driver. It could help save thousands of lives that are lost each year in car accidents. Vehicular communication networks will provide a wide range of applications with different characteristics. In detail, the information exchanged can be of two types, (i) periodic exchange of status messages among the vehicles in the direct communication range and (ii) safety messages triggered by a critical event and distributed in a geographical region. Roadside Communication units can provide drivers with information which help them in controlling the vehicle. These may be conventional vehicles driven by humans or they could even be autonomous vehicles. The system enables us to automatically monitor and/or control the actual speed of a vehicle relative to the legal speed limit of the geographic area (Schools, Colleges, Hospitals, etc.) the vehicle is located. In addition to this, the system is also capable of detecting an approaching landslide, earthquake or occurring one and transmitting pre safe warning. This vehicular communication system enables immediate payment system in which the vehicle can pay the tolls by the wireless fund transfer method. The proposed system is capable of measuring the level of fuel in the tank and send enquiry to know the nearest location of fuel station, then Wireless Communication equipped fuel station automatically gives the information about that location. Thus people are in need of this kind of system to safeguard their valuable lives.

\section{B. Objective}

The main objective of this paper is to communicate vehicle using wireless sensor network technology along with embedded system technology that enables interaction with the component or a device for effective functionality. That is, by enabling vehicles to communicate with one another, and their surrounding environment, it is hoped that key information can be shared in order to create a safer and more comfortable drive and also through wireless communication between the invehicle device and the roadside unit to enable fuel enquiry, toll gate payment, intelligent speed control and pre safe warning. 


\title{
International Journal of Advanced Research in Computer and Communication Engineering
}

\author{
Vol. 8, Issue 2, February 2019
}

\section{Scope}

The scope of the paper is to provide vehicle to vehicle communication that enable the user to know about the current situation of the road such as soil moisture level of the road so that user can be prevent from accident, fuel advice and search of nearby fuel station enquiry, speed control and the immediate toll payment system by wireless fund transfer method.

\section{LITERATURE SURVEY}

\section{A. Vehicle-To-Vehicle Safety Messaging in DSRC}

This paper studies the design of layer-2 protocols for a vehicle to send safety messages to other vehicles. The target is to send vehicle safety messages with high reliability and low delay. The communication is one-to-many, local, and geosignificant. The vehicular communication network is ad-hoc, highly mobile, and with large numbers of contending nodes. The messages are very short, have a brief useful lifetime, but must be received with high probability. For this environment, this paper explores the efficacy of rapid repetition of broadcast messages. This paper proposes several random access protocols for medium access control. The protocols are compatible with the Dedicated Short Range Communications (DSRC) multi-channel architecture. Analytical bounds on performance of the proposed protocols are derived. Simulations are conducted to assess the reception reliability and channel usage of the protocols. The sensitivity of the protocol performance is evaluated under various offered traffic and vehicular traffic flows. The results show the approach is feasible for vehicle safety messages in DSRC.

\section{B. Vehicle-To-Vehicle Wireless Communication Protocols for Enhancing Highway Traffic Safety}

This paper presents an overview of highway cooperative collision avoidance (CCA), which is an emerging vehicular safety application using the IEEE and ASTM-adopted Dedicated Short Range Communication (DSRC) standard. Along with a description of the DSRC architecture, the concept of CCA and its implementation requirements in the context of a vehicleto-vehicle wireless network, primarily at the Medium Access Control (MAC) and the routing layer. An overview is then provided to establish that the MAC and routing protocols from traditional Mobile Ad Hoc networks are not directly applicable for CCA and similar safety-critical applications. Specific constraints and future research directions are then identified for packet routing protocols used to support such applications in the DSRC environment. In order to further explain the interactions between CCA and its underlying networking protocols, an example of the safety performance of CCA using simulated vehicle crash experiments. The results from these experiments are also used to demonstrate the need for network data prioritization for safety-critical applications such as CCA.

\section{A Vehicle-To-Vehicle Communication Protocol for Cooperative Collision Warning}

This paper proposes a vehicle-to-vehicle communication protocol for cooperative collision warning. Emerging wireless technologies for vehicle-to-vehicle (V2V) and vehicle-to-roadside (V2R) communications such as DSRC are promising to dramatically reduce the number of fatal roadway accidents by providing early warnings. One major technical challenge addressed in this paper is to achieve low-latency in delivering emergency warnings in various road situations. Based on a careful analysis of application requirements, an effective protocol, comprising congestion control policies, service differentiation mechanisms and methods for emergency warning dissemination. Simulation results demonstrate that the proposed protocol achieves low latency in delivering emergency warnings and efficient bandwidth usage in stressful road scenarios.

D. Performance of Vehicle-To-Vehicle Communication using IEEE 802.11p in Vehicular Ad-Hoc Network Environment Traffic safety applications using vehicle-to-vehicle (V2V) communication is an emerging and promising area within the ITS environment. Many of these applications require real-time communication with high reliability. To meet a real-time deadline, timely and predictable access to the channel is paramount. The medium access method used in 802.11p, CSMA with collision avoidance, does not guarantee channel access before a finite deadline. The well-known property of CSMA is undesirable for critical communications scenarios. The simulation results reveal that a specific vehicle is forced to drop over $80 \%$ of its packets because no channel access was possible before the next message was generated. To overcome this problem, the use STDMA for real-time data traffic between vehicles. The real-time properties of STDMA are investigated by means of the highway road simulation scenario, with promising results.

\section{E. Vehicle-To-Vehicle and Road-Side Sensor Communication for Enhanced Road Safety}

This paper proposes a hybrid ITS safety architecture that combines vehicle-to-vehicle communication and vehicle-toroadside sensor communication. Opposed to dedicated roadside units, which require major investments for purchase, installation and maintenance, roadside wireless sensor and networking technology represents a cost-effective solution and 


\title{
International Journal of Advanced Research in Computer and Communication Engineering
}

\author{
Vol. 8, Issue 2, February 2019
}

can leverage the deployment of the system as a whole. Among the various services of the hybrid communication system, the paper introduces accident prevention and post-accident investigation. A system and protocol architecture is a fully distributed concept for efficient and secure storage of sensor data. For deployment, this architecture will likely be combined with an alternative approach using dedicated road-side units as a centralized network element for communication and data storage. The proposed system describes the main components (radio, networking and services, security). Finally, It describes the prototype implementation and experimental test bed featuring hardware and software platforms for vehicle on-board units and sensor nodes.

\section{SYSTEM ANALYSIS}

\section{A. Existing System}

The existing system in toll payment is either by open and close toll collection system which collects the toll manually by the toll collector and the other by electronic toll collection system which involves the unique number of the RFID FASTag affixed on the wind shield of the vehicle will be read by the readers fitted in the dedicated 'ETC' lanes of plazas and the toll will be deducted automatically without stopping the vehicle. The existing system in fuel enquiry is the system which can indicate that the fuel is low. The existing system in speed control is the system which is capable of indicating and restricting speed limit. There is no similar existing system in case of pre safe warning.

\section{B. Disadvantages of Existing System}

The existing system involves manual payment of the toll plazas which leads to the high traffic peak in order to pay the toll. This leads to more manpower and time waste for the vehicle drivers to wait in a huge queue. The other method is the electronic toll collection system involves the RFID can be expensive because of their batteries and the objects gets read when it is close to the toll plazas i.e., about 30 meter. The existing system in fuel enquiry is not capable of indicating nearest fuel station. The existing system in speed control is not capable of controlling the speed of the vehicle based on geographical locations.

\section{Proposed System}

The Proposed system involves Zigbee which is used instead of the RFID and it is a standardized protocol for Ultra Low Power Wireless Personal Area Networks (WPANs). Zigbee is an established set of specifications for wireless personal area networking (WPAN) i.e., digital radio connections between computers and related devices. Zigbee is targeted at radiofrequency (RF) applications which require a low data rate, long battery life, and secure networking. Zigbee is set of high level communication protocols based upon the specification produced by 802.15.4 - standard for wireless personal area networks (WPANs). A personal area network (PAN) is a computer network used for communication among computer devices (including telephones and personal digital assistants) close to one person. A wireless PAN consists of a dynamic group of less than 255 devices that communicate within a range of about 33 -feet. Zigbee has $10 \mathrm{kbps}$ to $115 \mathrm{kbps}$ data throughput, 10 to $75 \mathrm{~m}$ coverage range, up to 100 collected networks and up to 2 years of battery life on standard alkaline batteries. This system also provides the pre-safe warning by road safety and speed control of the vehicle. Pulse width modulation is used to calculate the speed of vehicle and based on that value motor is rotated in the vehicle. Today's, industries are increasingly demanding process automation in all sectors. Automation results into better quality, increased production and reduced costs. The variable speed drives, which sleeplessly control speed of D.C. Motors, are indispensable controlling elements in automation systems.

The existing speed control systems uses wired or wireless technology especially Infrared communication. The drawback with the IR communication is that: it is a point-to-point communication and the limited distance communication. So this limits the distance at which the motor is controlled and also need line of sight between the IR transmitter and the IR receiver. This leads to the lower performance of the variable speed drive. This paper overcomes the above stated drawbacks by providing a remote control operation to the motor speed control using RF technology. The advantage of the RF based system is that: it can provide a larger control distance over RF and no line of sight is needed. The RF based remote motor speed controller receives commands from a remote-host, compares them to the actual position, and calculates the desired motor drive level. The RF transmitter and receiver operate at the frequency of $433 \mathrm{MHz}$. The arduino microcontroller uses PWM technique to control the speed of the remotely positioned DC motor. This system is ideally suited for remotely positioned valves and machinery used in industrial applications. The road safety is detected by the soil moisture sensor such as earthquakes, landslides and soil eruption occurs in the driving root means, it will intimate the user and suggest the user to take another safer root. Fuel advice and the fuel status enquiry are given when the fuel is at the minimum level of about 20 percentages. 


\section{International Journal of Advanced Research in Computer and Communication Engineering}

Vol. 8, Issue 2, February 2019

\section{Advantages of Proposed System}

Driver assistance for fuel indication and automatic detection of nearby petrol bunk stations, E-payment for tollgate by installing intelligent system in the controllers of vehicle, Soil moisture detector to determine whether the transportation is suitable or not and Intel speed control of the vehicle are provided by the proposed system. In toll payment the battery power is saved by using zigbee instead of the RFID tag reader and zigbee can sense the vehicle up to (30-100) meters i.e., the vehicles are nearby toll plazas.

\section{E. Applications}

The proposed system has a variety of application in the field of automobile industry where wireless communication system has been implemented. The wireless communication includes vehicle-to-vehicle and vehicle-to-tollgate. Zigbee enables the wireless communication. The system can be implemented in four wheeler transport.

\section{SYSTEM DESIGN}

\section{A. System Architecture}

System architecture is the conceptual model that defines the structure, behaviour, and more views of a system. An architecture description is a formal description and representation of a system, organized in a way that supports reasoning about the structures and behaviours of the system. Figure 1 shows the NODE 1(vehicle) system architecture and Figure 2 shows the NODE 2 (roadside) system architecture.

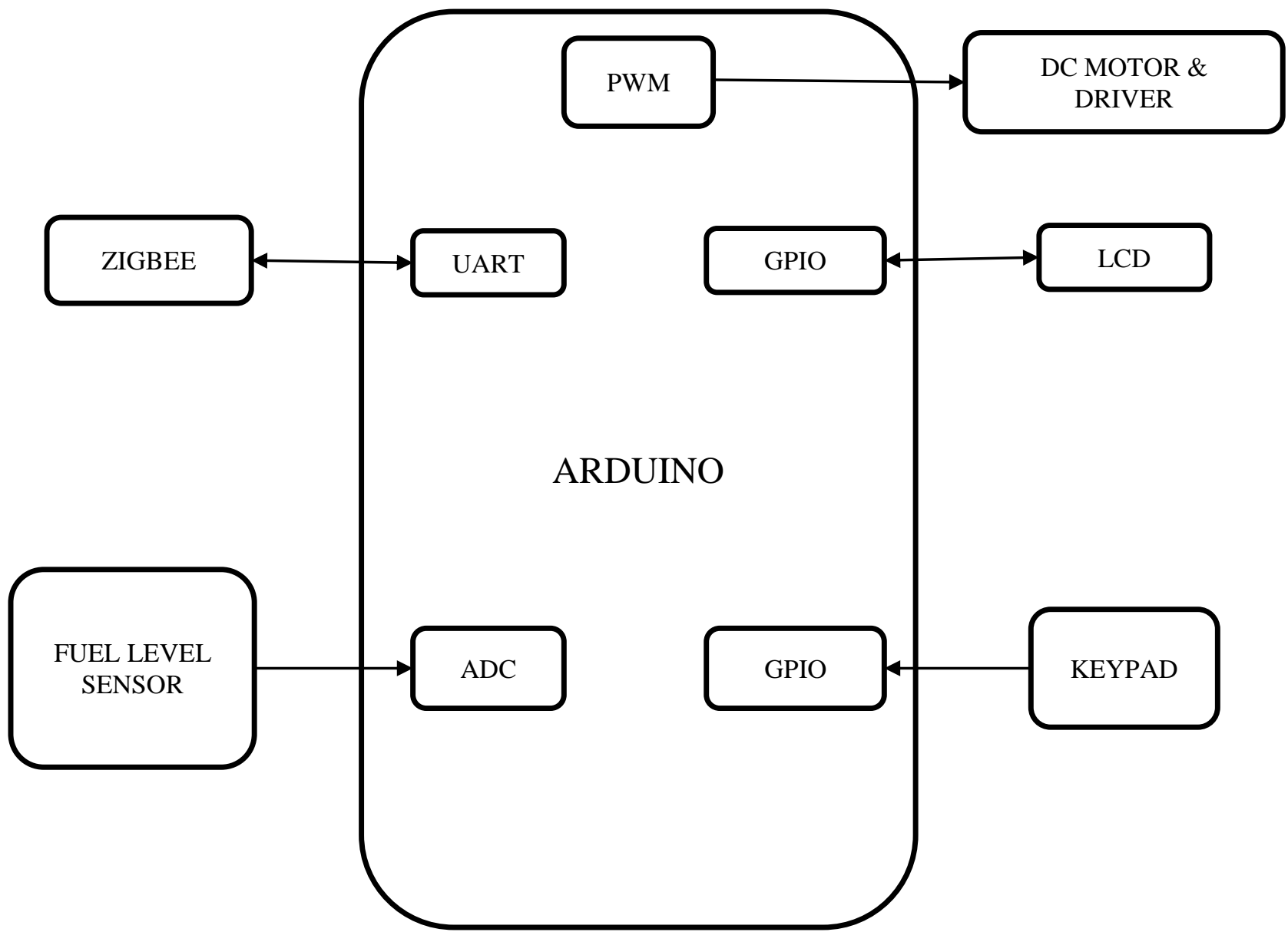

Fig. 1 Vehicle Node 


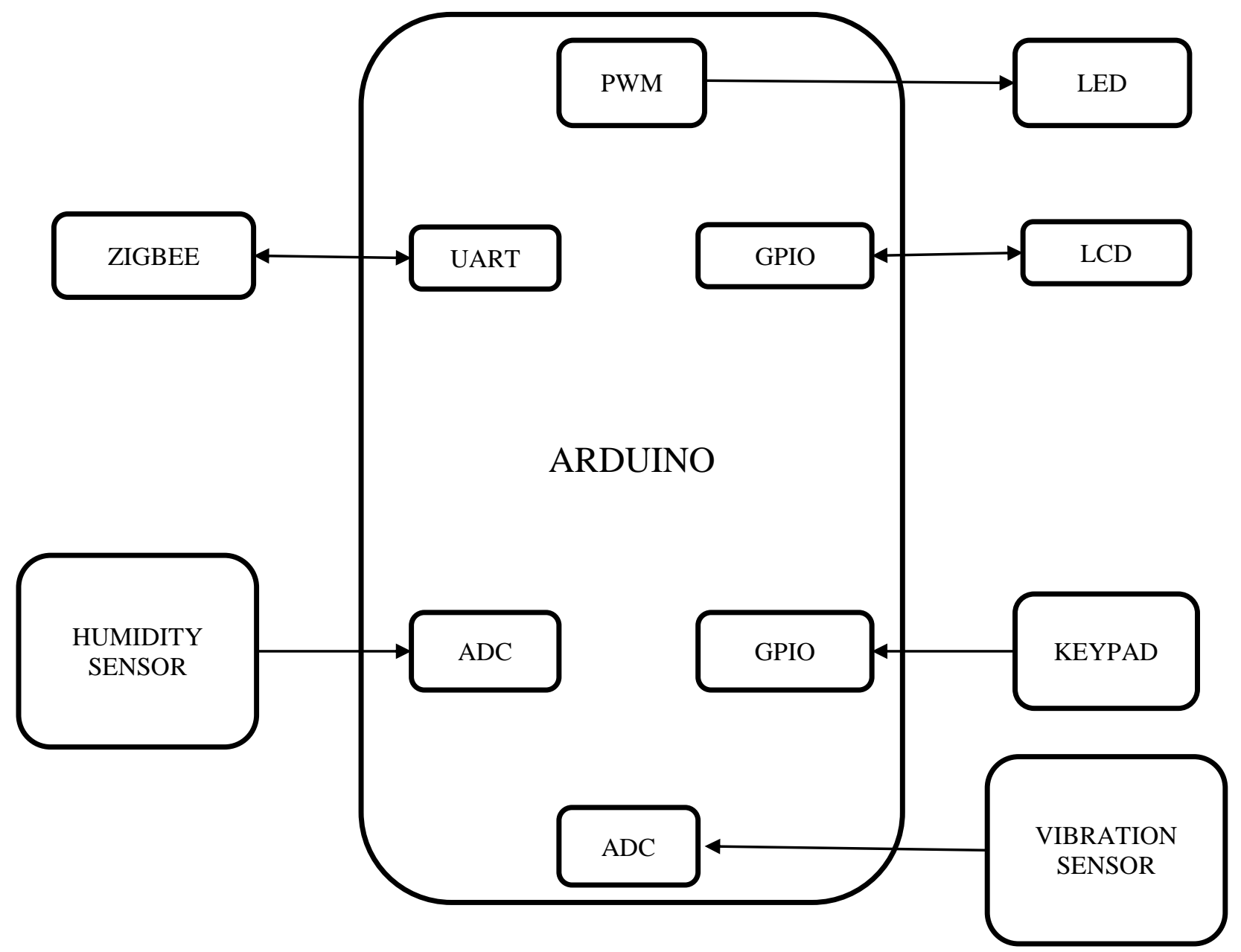

Fig. 2 Roadside Node

\section{1) Arduino:}

Arduino is an open source, computer hardware and software company, project, and user community that designs and manufactures microcontroller kits for building digital devices and interactive objects that can sense and control objects in the physical world. The project's products are distributed as open-source hardware and software, which are licensed under the GNU Lesser General Public License (LGPL) or the GNU General Public License (GPL), permitting the manufacture of Arduino boards and software distribution by anyone. Arduino boards are available commercially in preassembled form, or as do-it-yourself kits.

Arduino board designs use a variety of microprocessors and controllers. The boards are equipped with sets of digital and analog input/output (I/O) pins that may be interfaced to various expansion boards (shields) and other circuits. The boards feature serial communications interfaces, including Universal Serial Bus (USB) on some models, which are also used for loading programs from personal computers. The microcontrollers are typically programmed using a dialect of features from the programming languages $\mathrm{C}$ and $\mathrm{C}++$. In addition to using traditional compiler toolchains, the Arduino project provides an integrated development environment (IDE) based on the Processing language project.

The Arduino project started in 2003 as a program for students at the Interaction Design Institute Ivrea in Ivrea, Italy, aiming to provide a low-cost and easy way for novices and professionals to create devices that interact with their environment using sensors and actuators . Common examples of such devices intended for beginner hobbyists include simple robots, thermostats, and motion detectors. The name Arduino comes from a bar in Ivrea, Italy, where some of the founders of the 


\title{
International Journal of Advanced Research in Computer and Communication Engineering
}

\author{
Vol. 8, Issue 2, February 2019
}

project used to meet. The bar was named after Arduino of Ivrea, who was the margrave of the March of Ivrea and King of Italy from 1002 to 1014

2) Keypad:

The $4 \times 4$ matrix keypad is a general-purpose keypad. It consists of 16 switches arranged in 4 rows and 4 columns. It can connect to the MCU 8-bit port directly.

3) $L C D$ :

The working of sensors plays a major role in the effectiveness of the system. The output is being displayed in a LCD display. LCD (Liquid Crystal Display) screen is an electronic display module and find a wide range of applications. A 16x2 LCD display is very basic module and is very commonly used in various devices and circuits. These modules are preferred over seven segments and other multi segment LEDs. The reasons being: LCDs are economical; easily programmable; have no limitation of displaying special and even custom characters (unlike in seven segments), animations and so on.

4) Zigbee:

Zigbee as standardized protocol for Ultra Low Power Wireless Personal Area Networks(WPANs). Zigbee is an established set of specifications for wireless personal area networking (WPAN) i.e., digital radio connections between computers and related devices.

5) Fuel Level Sensor:

Fuel level sensor sense the fuel level of the vehicle and it intimate the fuel level in the fuel tank to the driver. Through zigbee communication the nearest fuel station can be identified and intimation can be send to the vehicle.

6) Humidity Sensor:

Humidity sensor sense the humidity level to check whether the humidity level is safety to drive the vehicle or not. If any circumstances arrive the user of the vehicle will be known by wireless sensor communication.

7) Vibration Sensor:

Vibration sensor sense the vibration level to check whether there is earthquake or not. If any circumstances arrive the user of the vehicle will be known by wireless sensor communication.

\section{SYSTEM IMPLEMENTATION}

System implementation is the process of defining how the information system should be built (i.e., physical system design), ensuring that the information system is operational and used, ensuring that the information system meets quality standard (i.e., quality assurance). The system is implemented using Embedded C, Arduino IDE, Android Studio and Proteus.

\section{A. Arduino IDE}

The Arduino project provides the Arduino Integrated Development Environment (IDE), which is a cross-platform application written in the programming language Java. It originated from the IDE for the languages Processing and Wiring . It includes a code editor with features such as text cutting and pasting, searching and replacing text, automatic indenting, brace matching, and syntax highlighting, and provides simple one-click mechanisms to compile and upload programs to an Arduino board. It also contains a message area, a text console, a toolbar with buttons for common functions and a hierarchy of operation menus.

\section{B. Android Studio}

Android Studio is the official integrated development environment (IDE) for Google's Android operating system, built on JetBrains' IntelliJ IDEA software and designed specifically for Android development. It is available for download on Windows, macOS and Linux based operating systems. It is a replacement for the Eclipse Android Development Tools (ADT) as the primary IDE for native Android application development.

\section{Proteus}

Proteus (PROcessor for TExt Easy to USe) is a fully functional, procedural programming language created in 1998 by Simone Zanella. Proteus incorporates many functions derived from several other languages: C, BASIC, Assembly, Clipper/dBase; it is especially versatile in dealing with strings, having hundreds of dedicated functions; this makes it one of the richest languages for text manipulation. Proteus owes its name to a Greek god of the sea (Proteus), who took care of Neptune's crowd and gave responses; he was renowned for being able to transform himself, assuming different shapes. Transforming data from one form to another is the main usage of this language. 


\title{
IJARCCE
}

\section{International Journal of Advanced Research in Computer and Communication Engineering}

\author{
Vol. 8, Issue 2, February 2019
}

\section{VI.MODULES}

The proposed system consists of four main modules. They are:

$$
\begin{array}{ll}
- & \text { Fuel advice and enquiry } \\
\text { - } & \text { Toll collection system } \\
\text { - } & \text { Pre safe warning } \\
& \text { Intelligent speed control }
\end{array}
$$

\section{A. Fuel Advice and Enquiry}

The technology is used to develop a more accurate way to measure the level in the fuel tank in vehicles. The fuel level should be displayed for the vehicle so that the driver will be aware if the fuel level is too low. Now the intelligent system can send the enquiry to know the nearest location of fuel station, then the Wireless Communication equipped fuel station automatically gives the information about that location through mobile application.

\section{B. Toll Collection System}

Through wireless communication between the in-vehicle device and the toll gate antenna, the cars are able to drive through toll gates without stopping (at speeds below approximately $20 \mathrm{~km} / \mathrm{hr}$ ). Tolls are payable in arrears by the wireless fund transfer method.

\section{Pre Safe Warning}

Landslides occur mainly due to heavy rainfall experienced by Landslide Hazard Zones during the monsoon season and sometimes as an aftermath of an earthquake. The solution this device gives is based upon the concept of low cost wireless sensor networks. WSN method uses a sensor network consisting of sensor columns. The sensor node consists of a MEMS acceleration sensor and a soil moisture sensor, a low power data processing unit (microcontroller) and wireless communication unit. If it detects an approaching landslide or an occurring one, the host intelligent system transmits emergency information. The warning is triggered by vehicles ahead reaching the area.

\section{Intelligent speed control}

This method for automatically monitoring and/or controlling the actual speed of a non-emergency vehicle relative to the legal speed limit of the geographic area the vehicle is located in.

\section{RESUlts}

Proteus simulation results always match with the original results (both theoretically and experimentally) it is tough to implement the original setup with all these hardware. Though implemented experimentally it is very expensive but the completion can be done soon.

Consider the vehicles are moving in different directions and with different speed. There fuel is module by fuel sensor. This will hold the information about the fuel level in the vehicle and it can also sense the nearest fuel station. The next module is the soil moisture. This module is done using soil moisture sensor which detect the soil moisture level and intimate the status to the vehicle through wireless sensor communication (WSN). The toll plaza module smoothen the payment using WFTM (wireless fund transfer method). Each module will have an attribute setting those and all have to be defined while creating the system.

This contains the code for the random modules for the system. In this the state variables are initialized and the status is obtained for each variables. Here it checks for the module status and then parse the module information one by one. After parsing the next line decides which action is to be performed. Finally it creates action for each and every signal.

The modules which the proposed system is designed are used to improve the road safety and immediate toll payment. The final product says that if as system can work successful in this environment it can work in all environment. By implementing this paper the road accident can be reduced and road safety will be improved and pass the toll will be easily done without stopping the vehicle on the toll plaza and the life of people can be saved by providing pre safe warning. If this project comes to the real life it will be more use full for the people as well the government and to the industries and it is used to prevent the accident that occur due to the earthquake, landslides and also by the people who use vehicle to smooth pass of the toll plaza. The system is a cost effective as compared to life of the people. Thus the system is an effective and a reliable system. 


\title{
International Journal of Advanced Research in Computer and Communication Engineering
}

\author{
Vol. 8, Issue 2, February 2019
}

\section{CONCLUSION AND FUTURE ENHANCEMENT}

\section{A. Conclusion}

The system is a safe and a reliable system that can prevent the accident from being occurred through pre safe warning and the road safety. The system could enable a safer driving environment as compared to the other manual driving environment. This system could enables pre safe waring and immediate toll payment, fuel level and fuel station intimation, MEMs vibration and soil moisture level. The system has a higher efficiency rate as compared to the existing system in the way that the proposed system does not produce much heat as compared to existing and hence the malfunction is minimum. The proposed system uses light weight components therefore the space and weight of the system is not much as compared to the existing system. The system helps in efficient working of the vehicle to vehicle communication with Zigbee and immediate toll payment combined with the Wireless Fund Transfer Method (WFTM). The system has a highly reliable and user friendly system which can interact with the user to deliver the warning and provide necessary notification.

\section{B. Future Enhancement}

The system could be further implemented in a real vehicle and the working can be checked for the efficiency. Currently the work is done using wired approach areas and which may be completely adapted to wireless environment.

\section{REFERENCES}

[1]. A. Zemlianov and G. de Veciana, "Capacity of ad hoc wireless networks with infrastructure support," IEEE J. Sel. Areas Commun., vol. 23, no. 3, pp. 657-667, Mar. 2005.

[2]. C. Lochert, B. Scheuermann, M. Caliskan, andM.Mauve, "The feasibility of information dissemination in vehicular ad-hoc networks," in Proc. 4th Annu. Conf. Wireless Demand Netw. Syst. Services, Jan. 2007, pp. 92-99.

[3]. N. Wisitpongphan, F. Bai, P. Mudalige, V. Sadekar, and O. K. Tonguz, "Routing in sparse vehicular ad hoc wireless networks," IEEE J. Sel. Areas Commun., vol. 25, no. 8, pp. 1538-1556, Oct. 2007.

[4]. P. Li, X. Huang, Y. Fang, and P. Lin, “Optimal placement of gateways in vehicular networks," IEEE Trans. Veh. Technol., vol. 56, no. 6, pp. 34213430, Nov. 2007.

[5]. M. Nekoui, A. Eslami, and H. Pishro-Nik, "The capacity of Vehicular Ad Hoc Networks with infrastructure," in Proc. 6th Int. Symp. WiOPT, Apr. 2008, pp. 267-272

[6]. S. Yousefi, E. Altman, R. Elazouzi, andM. Fathy, "Improving connectivity in vehicular ad-hoc networks: An analytical study," Comput. Commun., vol. 31, no. 9, pp. 1653-1659, Jun. 2008.

[7]. C. Lochert, B. Scheuermann, C. Wewetzer, A. Luebke, and M. Mauve, "Data aggregation and roadside unit placement for a VANET traffic information system," in Proc. 5th ACM Int'1 Workshop Veh. Internetworking, San Francisco, CA, USA, Sep. 2008, pp. 58-65.

[8]. A. Kchiche and F. Kamoun, "Centrality-based access-points deployment for vehicular networks," in Proc. IEEE 17th Int. Conf. Telecommun., 2010, pp. 700-706.

[9]. K. Mershad and H. Artail, "Using RSUs as delegates for pervasive access to services in vehicle ad hoc networks," in Proc. 17th IEEE Int. Conf. Telecommun., Doha, Qatar, Apr. 2010, pp. 790-797.

[10]. S. C. Ng, W. Zhang, Y. Zhang, Y. Yang, and G. Mao, "Analysis of access and connectivity probabilities in infrastructure-based vehicular relay networks," in Proc. IEEE WCNC, Apr. 2010, pp. 1-6.

[11]. Y. Yang, Z. Mi, J. Y. Yang, and G. Liu, "A model based connectivity improvement strategy for vehicular ad hoc networks," in Proc. IEEE 72nd VTC, Sep. 2010, pp. 1-5.

[12]. B. Liu, B. Khorashadi, D. Ghosal, C.-N. Chuah, and M. H. Zhang, "Assessing the VANET's local information storage capability under different traffic mobility," in Proc. IEEE INFOCOM, 2010, pp. 1-5.

[13]. B. Aslam and C. C. Zou, "Optimal roadside units placement along highways," in Proc. IEEE CCNC, Jan. 2011, pp. 814-815.

[14]. S. Busanelli, G. Ferrari, V. A. Giorgio, and N. Iotti, "Comparative investigation of single-hop and multi-hop broadcast strategies for information dissemination in VANETs," in Proc. 11th Int. Conf. ITST, Aug. 2011, pp. 738-743.

[15]. W. Zhang, Y. Chen, Y. Yang, X. Wang, Y. Zhang, X. Hong, and G. Mao, "Multi-hop connectivity probability in infrastructure-based vehicular networks," IEEE J. Sel. Areas Commun., vol. 30, no. 4, pp. 740-747, May 2012. 